\title{
Comportamentos de proteção contra COVID-19 entre adultos e idosos brasileiros que vivem com multimorbidade: iniciativa ELSI-COVID-19
}

\author{
Protective behaviors for COVID-19 among \\ Brazilian adults and elderly living with \\ multimorbidity: the ELSI-COVID-19 initiative
}

\section{Comportamientos de protección contra la COVID-19 entre adultos y ancianos brasileños que viven con multimorbilidad: la iniciativa ELSI-COVID-19}

Sandro Rodrigues Batista 1,2 Ana Sara Semeão de Souza 3 Januse Nogueira 4 Fabiola Bof de Andrade 5

Elaine Thumé 6 Doralice Severo da Cruz Teixeira 7 Maria Fernanda Lima-Costa 5,8 Luiz Augusto Facchini 9 Bruno Pereira Nunes 6

doi: 10.1590/0102-311X00196120

\section{Resumo}

Objetivou-se medir a ocorrência de comportamentos de proteção contra a COVID-19 e fatores sociodemográficos segundo a ocorrência de multimorbidade na população brasileira com 50 anos ou mais de idade. Foram utilizados dados de inquérito telefônico entre participantes do ELSI-Brasil (Estudo Longitudinal da Saúde dos Idosos Brasileiros), conduzido entre maio e junho de 2020. Avaliou-se o uso de medidas de prevenção não farmacológica para COVID-19, motivos para sair de casa segundo a presença de multimorbidade e variáveis sociodemográficas. Participaram do estudo 6.149 pessoas. Multimorbidade foi mais frequente no sexo feminino, em casados, na faixa etária 50-59 anos de idade e em moradores da zona urbana. A maior parte da população saiu de casa entre uma e duas vezes na última semana, percentual que aumentou segundo o número de morbidades (22,3\% sem morbidades e 38\% com multimorbidade). Sair de casa todos os dias teve menor ocorrência entre indivíduos com multimorbidade (10,3\%), e 9,3\% saíram de casa na última semana para obter atendimento de saúde. Higienização de mãos (>98\%) e sempre usar máscara ao sair de casa (> 96\%) foram hábitos quase universais. $\mathrm{Ob}$ servou-se maior adesão ao isolamento social entre as mulheres com multimorbidade quando comparadas com os homens ( $R P=1,49$; IC95\%: 1,23-1,79); esta adesão aumentou proporcionalmente com a idade e inversamente ao nível de escolaridade. O comportamento de proteção em pessoas com multimorbidade parece ser maior em relação aos demais, embora questões relacionadas ao isolamento social e cuidado em saúde mereçam ser destacadas. Esses achados podem ser úteis na customização de estratégias de enfrentamento atual da pandemia.

Multimorbidade; COVID-19; Comportamento; SARS-CoV-2; Doença Crônica

\section{Correspondência}

S. R. Batista

Departamento de Clínica Médica, Faculdade de Medicina, Universidade Federal de Goiás.

Av. Primeira Avenida s/n, Goiânia, GO 74605-020, Brasil. sandrorbatista@gmail.com

1 Faculdade de Medicina, Universidade Federal de Goiás, Goiania, Brasil.

2 Secretaria de Estado da Saúde de Goiás, Goiânia, Brasil. 3 Instituto de Medicina Social, Universidade do Estado do Rio de Janeiro, Rio de Janeiro, Brasil.

4 Universidade Federal de Campina Grande, Campina Grande, Brasil.

5 Instituto René Rachou, Fundação Oswaldo Cruz, Belo Horizonte, Brasil.

6 Departamento de Enfermagem, Universidade Federal de Pelotas, Pelotas, Brasil.

7 Faculdade de Saúde Pública, Universidade de São Paulo, São Paulo, Brasil.

8 Programa de Pós-graduação em Saúde Púbica, Universidade Federal de Minas Gerais, Belo Horizonte, Brasil.

${ }^{9}$ Faculdade de Medicina, Universidade Federal de Pelotas, Pelotas, Brasil. 


\section{Introdução}

Nos dias atuais, a sociedade e os sistemas de saúde em todo o mundo têm sido desafiados devido ao surgimento de uma nova síndrome respiratória aguda grave do coronavírus 2 (SARS-CoV-2). Em dezembro de 2019, na província de Hubei na China, foram relatados os primeiros casos de doença de coronavírus 2019 (COVID-19) com posterior propagação mundial chegando-se ao status de pandemia em 7 de abril de 2020 1. Até 2 de julho de 2020 já se somavam mais de 10 milhões e setecentos mil casos confirmados de COVID-19 em 216 países e territórios atingindo um total de mais de meio milhão de óbitos em decorrência da doença. No Brasil, o primeiro caso foi confirmado em 26 de fevereiro de 2020 e até o início do mês de julho já havia quase 1 milhão e quinhentos mil casos e mais de 60 mil óbitos (World Health Organization. Who coronavirus disease (COVID-19) dashboard. https:// covid19.who.int/).

Embora, a princípio, cause uma doença respiratória, em grande parte casos assintomáticos ou oligossintomáticos, o SARS-CoV-2 pode causar síndrome respiratória grave e acometer outros sistemas corpóreos, como no caso da MERS-CoV (middle east respiratory syndrome) ${ }^{2}$. As formas severas de COVID-19 têm maior probabilidade de se desenvolver em pessoas mais velhas e naquelas portadoras de doenças crônicas prévias 3,4,5,6,7. Nesse sentido, a ocorrência da multimorbidade, ou seja, a presença de duas ou mais morbidades crônicas, é um fator de risco importante a ser considerado, pois, por si só, é um preditor de risco de morte 5,8. Estudos com a população brasileira demonstram prevalência de $22,2 \%$ ( $\geq 2$ morbidades) e 10,2\% ( $\geq 3$ morbidades) em indivíduos acima de 18 anos 9 . Entretanto, estima-se que $67,8 \%$ dos brasileiros acima de 50 anos vivem com multimorbidade 10 .

Multimorbidade e COVID-19 têm importante relação com iniquidades em saúde, de modo que indivíduos com piores condições socioeconômicas tendem a ser mais afetados 11,12,13. Alguns autores têm revisitado o termo sindemia (originalmente usado nos Estados Unidos na década de $1990 \mathrm{em}$ discussões sobre as relações entre HIV/aids, uso de substâncias e violência) numa referência ao cenário atual de COVID-19, desigualdades em morbidades crônicas e determinantes sociais da saúde e seu impacto nas iniquidades de taxas de infecção e mortalidade por COVID-19 14. Soma-se aqui o fato de que o Brasil é um dos países mais desiguais do mundo e, nos últimos quatro anos, apresentou um aumento de $33 \%$ na pobreza e a tendência mais sustentada no aumento da desigualdade de renda já registrada na história do país 15 . A robustez dessa associação foi observada nos últimos meses, com o deslocamento do epicentro da pandemia no país para os estados da regiões Norte e Nordeste, gerando impacto desigual, com taxas desproporcionalmente altas de infecção e letalidade por COVID-19 16,17.

Como medidas para mitigar o surto de COVID-19, muitos países decretaram ações para controle de movimentação social e proteção dos grupos de risco na perspectiva de que o cumprimento de medidas preventivas, principalmente entre os idosos, minimize o número de casos e mortes 18,19,20. No Brasil, o Ministério da Saúde declarou o estado de transmissão comunitária da COVID-19 em todo o território nacional em 20 de março de 2020 21, objetivando promover medidas não farmacológicas, sobretudo promover o distanciamento social e evitar aglomerações. Além disso, recomendou às pessoas com mais de 60 anos de idade o distanciamento social, restringindo seus deslocamentos para realização de atividades estritamente necessárias, com uso de máscaras e higienização das mãos de forma adequada.

Assim, frente ao contexto de elevada prevalência de morbidades crônicas e multimorbidade, principalmente em brasileiros mais velhos, associada às relevantes iniquidades sociais e ao reconhecimento da transmissão comunitária da COVID-19, objetivou-se, com o presente estudo, medir a ocorrência de comportamentos de proteção contra a COVID-19 e fatores sociodemográficos segundo a ocorrência de multimorbidade na população com 50 anos ou mais de idade. 


\section{Métodos}

\section{Amostra}

Foram utilizados dados de indivíduos participantes do Estudo Longitudinal da Saúde dos Idosos Brasileiros (ELSI-Brasil) 22. Trata-se de estudo longitudinal de base populacional iniciado em 2015-2016, delineado para representar a população brasileira com 50 anos ou mais. A segunda onda do ELSI-Brasil foi iniciada em agosto de 2019, mas interrompida em 17 de março de 2020, em decorrência da epidemia da SARS-CoV-2, sendo possível a obtenção de dados de 9.177 pessoas.

Neste contexto, foi proposto, de forma complementar, inquérito telefônico especificamente para avaliações referentes à COVID-19 nessa população. Todos os participantes da segunda onda do ELSI-Brasil foram elegíveis para o inquérito telefônico, denominado iniciativa ELSI-COVID-19 e realizado por meio de entrevista com duração média de cinco minutos. Avaliou-se a utilização de comportamentos de proteção não farmacológica para COVID-19 (isolamento domiciliar, uso de máscaras e higienização das mãos), motivos para sair de casa, apoio para compra de alimentos e medicamentos, diagnóstico médico para a COVID-19 e realização de testes confirmatórios, bem como uso de serviços de saúde e questões associadas à saúde mental. As entrevistas telefônicas foram realizadas entre os dias 26 de maio e 8 de junho de 2020, e maiores detalhes metodológicos da iniciativa ELSI-COVID-19 podem ser acessados em publicação específica 23.

Dessa forma, obteve-se informação de 6.149 participantes (67\% da segunda onda do ELSI-Brasil). Desse total, 27,8\% foram respondidas por um informante. As características dos participantes foram semelhantes àquelas da população brasileira com 50 anos ou mais no que se refere a todas as características sociodemográficas analisadas.

\section{Variáveis}

A adoção de comportamentos de proteção contra a COVID-19 foi considerada a variável desfecho para esse estudo. Para tal, foram avaliados: saída do domicílio (periodicidade e motivos); utilização de máscaras de proteção ao sair do domicílio e lavagem de mãos ou uso de álcool em gel para higienização. Os motivos para sair do domicílio foram categorizados em essenciais (comprar remédios ou alimentos/trabalhar/pagar contas/obter atendimento de saúde) e não essenciais (encontrar amigos e familiares/fazer atividade física/outras).

Para a avaliação de multimorbidade, foi realizada a contagem simples das seguintes morbidades 24: (1) hipertensão; (2) acidente vascular encefálico; (3) infarto agudo do miocárdio; (4) angina; (5) insuficiência cardíaca; (6) doença renal crônica; (7) doença de Alzheimer; (8) doença de Parkinson; (9) doença pulmonar obstrutiva crônica, enfisema ou bronquite crônica; (10) diabetes; (11) artrite; (12) asma; (13) câncer; (14) depressão; e (15) obesidade. Todas as morbidades tiveram o mesmo peso (1) na contagem total de morbidades e foram obtidas por autorrelato baseado em diagnóstico médico, exceto para obesidade, a qual foi avaliada por meio de aferição do índice de massa corporal (IMC), obtido mediante a divisão do peso pela altura ao quadrado (ambos média de duas medidas), e categorizada considerando os seguintes pontos de corte IMC $\geq 30 \mathrm{~kg} / \mathrm{m}^{2}$ e IMC $\geq 27 \mathrm{~kg} / \mathrm{m}^{2}$ para indivíduos com menos de 60 e $\geq 60$ anos de idade 25,26 respectivamente. Dessa forma, foram consideradas as seguintes categorias: sem morbidade crônica, presença de pelo menos uma morbidade crônica e multimorbidade ( $\geq 2$ morbidades). Indivíduos que não sabiam a resposta ou não responderam a alguma pergunta sobre as morbidades aferidas $(1,3 \%)$ foram considerados sem a morbidade.

Sexo (feminino, masculino), idade em anos completos (50-59, 60-69, 70-79, $\geq 80$ ) e escolaridade (nunca estudou, 1-4, 5-8, $\geq 9$ anos de estudo) foram as exposições principais. Cor da pele/raça autorreferida (branca, negra/parda, amarela e indígena), situação conjugal (solteiro, casado e viúvo), número de moradores na casa $(1,2, \geq 3)$, zona de residência (urbana, rural), região geopolítica do Brasil (Norte, Nordeste, Centro-oeste, Sudeste e Sul) e número de moradores no domicílio foram consideradas covariáveis. 


\section{Análise estatística}

As análises foram realizadas no software Stata SE 15.0 (https://www.stata.com) e incluíram cálculo de prevalência (\%) e intervalo de 95\% de confiança (IC95\%). Foram estimadas as prevalências dos comportamentos de proteção segundo o número de morbidades crônicas. Realizou-se análise multivariável, usando-se a regressão de Poisson, considerando o ajuste "para trás" (backward elimination) a fim de considerar somente as variáveis com possível efeito de confusão na análise. Essa análise também levou em conta a estratificação pelo número de morbidades $(0,1, \geq 2)$ para avaliar a associação entre o desfecho não sair de casa e sexo, idade e escolaridade. Para cada estrato, todas as demais variáveis de ajuste (cor da pele, número de moradores, zona de residência e região geopolítica) foram incluídas no modelo em um único nível hierárquico. Após o primeiro ajuste, identificou-se a variável com maior valor de $\mathrm{p}>0,20$, sendo excluída do próximo ajuste; esse processo foi repetido enquanto permaneceram, no modelo, as variáveis com valor de $\mathrm{p}>0,20$. As razões de prevalência (RP) e os intervalos de confiança apresentados na tabela refletem o ajuste para todas as variáveis com valor de $\mathrm{p}<0,20$. Associações com IC95\% sem incluir a unidade 1 foram consideradas estatisticamente significativas. Os parâmetros amostrais e pesos dos indivíduos especificamente derivados para o inquérito telefônico foram considerados em todas as análises.

\section{Aspectos éticos}

A iniciativa ELSI-COVID-19 foi aprovado pelo Comitê de Ética e Pesquisa da Fundação Oswaldo Cruz (Fiocruz, Minas Gerais - CAAE: 34649814.3.0000.5091).

\section{Resultados}

Participaram do estudo 6.149 pessoas, representando um total de 54.072.159 indivíduos com 50 anos ou mais de idade no Brasil. Dentre aqueles que relataram multimorbidade (45,8\%), a maioria era do sexo feminino (61,6\%), casado(a) (58,9\%), tinha entre 50 e 59 anos de idade $(36,7 \%)$, raça/cor da pele branca (52,2\%) e eram residentes na região Sudeste (42,3\%). Quanto à escolaridade, 40,6\% tinham nove anos ou mais de estudo, $87,1 \%$ moravam em zona urbana e mais da metade dividiam o domicílio com dois ou mais moradores (Tabela 1).

\section{Tabela 1}

Características da população de estudo segundo presença de multimorbidade. Inquérito telefônico iniciativa ELSI-COVID-19, 2020.

\begin{tabular}{|c|c|c|c|}
\hline \multirow[t]{3}{*}{ Variáveis } & \multicolumn{2}{|c|}{ Sem multimorbidade } & \multirow{3}{*}{$\begin{array}{c}\text { Com multimorbidade } \\
\geq 2 \text { morbidades } \\
\%(\text { IC } 95 \%)\end{array}$} \\
\hline & Sem morbidade & 1 morbidade & \\
\hline & $\%($ IC95\%) & $\%(I C 95 \%)$ & \\
\hline \multicolumn{4}{|l|}{ Sexo } \\
\hline Feminino & $47,4(40,6-54,3)$ & $48,9(44,3-53,4)$ & $61,6(57,0-66,0)$ \\
\hline Masculino & $52,5(45,6-59,3)$ & $51,1(46,5-55,6)$ & $38,3(33,9-42,9)$ \\
\hline \multicolumn{4}{|l|}{ Idade (anos) } \\
\hline $50-59$ & $63,1(56,0-69,8)$ & $49,4(44,3-54,7)$ & $36,7(30,1-43,9)$ \\
\hline $60-69$ & $23,1(18,7-28,2)$ & $27,0(24,0-30,3)$ & $33,7(30,4-37,3)$ \\
\hline $70-79$ & $9,1(6,5-12,7)$ & $15,9(12,9-19,5)$ & $20,1(16,0-25)$ \\
\hline$\geq 80$ & $4,4(2,9-6,6)$ & $7,4(5,5-9,8)$ & $9,2(6,9-12,2)$ \\
\hline
\end{tabular}

(continua) 
Tabela 1 (continuação)

\begin{tabular}{|c|c|c|c|}
\hline \multirow[t]{3}{*}{ Variáveis } & \multicolumn{2}{|c|}{ Sem multimorbidade } & \multirow{3}{*}{$\begin{array}{c}\text { Com multimorbidade } \\
\geq 2 \text { morbidades } \\
\% \text { (IC95\%) }\end{array}$} \\
\hline & Sem morbidade & 1 morbidade & \\
\hline & $\%(I C 95 \%)$ & $\%($ IC95\%) & \\
\hline \multicolumn{4}{|l|}{ Raça/Cor } \\
\hline Brancos & $56,1(45,3-66,2)$ & $49,6(41,1-58,0)$ & $52,2(44,1-60,2)$ \\
\hline Pretos/Pardos & $43,1(33,0-53,7)$ & $49,6(41,4-57,9)$ & $47,0(38,9-55,3)$ \\
\hline Amarelos & $0,7(0,1-3,0)$ & $0,1(0,0-1,4)$ & $0,3(0,0-2,8)$ \\
\hline Indígenas & $0,1(0,0-0,4)$ & $0,5(0,1-2,8)$ & $0,3(0,1-0,6)$ \\
\hline \multicolumn{4}{|l|}{ Escolaridade (anos) } \\
\hline Nunca estudou & $5,6(3,9-8,1)$ & $7,7(6,0-9,8)$ & $7,5(5,6-9,9)$ \\
\hline $1-4$ & $16,5(13,2-20,4)$ & $20,6(17,7-23,9)$ & $22,6(19,9-25,6)$ \\
\hline $5-8$ & $25,2(21,0-29,9)$ & $28,0(23,6-32,9)$ & $29,1(24,9-33,8)$ \\
\hline$\geq 9$ & $52,5(45,0-59,8)$ & $43,5(37,7-49,5)$ & $40,6(34,5-47,0)$ \\
\hline \multicolumn{4}{|l|}{ Situação conjugal } \\
\hline Solteiro(a) & $30,7(24,5-37,6)$ & $24,4(19,8-29,8)$ & $22,9(17,0-30,1)$ \\
\hline Casado(a) & $59,4(51,3-67,0)$ & $60,8(54,9-66,3)$ & $58,9(53,3-64,2)$ \\
\hline Viúvo(a) & $9,9(7,5-12,9)$ & $14,7(11,8-18,1)$ & $18,0(14,9-21,6)$ \\
\hline \multirow{2}{*}{\multicolumn{4}{|c|}{$\begin{array}{l}\text { Número de moradores } \\
\text { no domicílio }\end{array}$}} \\
\hline & & & \\
\hline 1 & $23,6(16,1-33,1)$ & $22,1(17,7-27,2)$ & $23,9(18,6-30,1)$ \\
\hline 2 & $37,8(32,0-43,9)$ & $38,4(33,8-43,1)$ & $40,4(36,2-44,6)$ \\
\hline$\geq 3$ & $38,5(30,6-47,1)$ & $39,4(32,9-46,4)$ & $35,6(30,2-41,5)$ \\
\hline \multicolumn{4}{|l|}{ Zona de moradia } \\
\hline Urbana & $89,9(85,6-92,9)$ & $85,5(78,6-90,4)$ & $87,1(82,8-90,5)$ \\
\hline Rural & $10,1(7,0-14,3)$ & $14,4(9,5-21,3)$ & $12,8(9,4-17,1)$ \\
\hline \multicolumn{4}{|l|}{ Região } \\
\hline Norte & $3,4(1,0-10,7)$ & $6,7(2,4-17,1)$ & $7,7(1,7-27,9)$ \\
\hline Nordeste & $28,9(16,1-46,4)$ & $28,5(18,2-41,6)$ & $24,9(16,3-36,0)$ \\
\hline Sudeste & $40,0(25,4-56,7)$ & $37,9(26,4-51,0)$ & $42,3(28,7-57,1)$ \\
\hline Sul & $19,8(7,4-43,3)$ & $15,4(7,4-29,5)$ & $14,2(7,5-25,4)$ \\
\hline Centro-oeste & $7,6(2,7-19,7)$ & $11,2(5,1-22,9)$ & $10,8(5,2-21,0)$ \\
\hline
\end{tabular}

IC95\%: intervalo de 95\% de confiança.

A prevalência de medidas de proteção contra a COVID-19, na população de estudo, segundo a presença de morbidades é demonstrada na Tabela 2. De forma geral, a maior parte da população saiu de casa entre uma e duas vezes na última semana ou não saiu de casa. A prevalência de pessoas que não saíram de casa aumentou segundo o número de morbidades, passando de $22,3 \%$ entre aqueles sem nenhuma morbidade para $38 \%$ entre os indivíduos com multimorbidade. Sair de casa todos os dias foi mais prevalente entre aqueles sem nenhuma morbidade $(21,1 \%)$ e menor entre indivíduos com multimorbidade (10,3\%). Os principais motivos para sair de casa foram a compra de remédios ou alimentos, trabalho e pagamento de contas, independente da classificação de morbidades. Entretanto, entre os indivíduos com multimorbidade, 9,3\% relataram ter saído de casa na última semana para obter atendimento de saúde. Já entre aqueles sem nenhuma morbidade, 9,1\% relataram ter saído de casa para encontrar amigos ou familiares.

Sempre usar máscara ao sair de casa foi maior que $96 \%$, independente do número de morbidades. No entanto, entre os indivíduos com multimorbidade, a prevalência de não uso ou de uso às vezes é menor em relação àqueles sem ou com uma morbidade. Assim, como o uso de máscara, a prevalência de lavagem das mãos ou uso de álcool em gel foi de, aproximadamente, $98 \%$ na população em geral, sendo percentualmente maior entre aqueles com multimorbidade (Tabela 2).

Em relação à escolaridade e sair de casa na última semana, pessoas com menor escolaridade ficaram mais em casa em relação às categorias mais altas de escolaridade, independente da classificação 
Tabela 2

Prevalência de medidas de proteção contra COVID-19 segundo presença de multimorbidade. Inquérito telefônico iniciativa ELSI-COVID-19, 2020.

\begin{tabular}{|c|c|c|c|}
\hline \multirow[t]{2}{*}{ Variáveis } & \multicolumn{2}{|c|}{ Sem multimorbidade } & \multirow{2}{*}{$\begin{array}{c}\text { Com multimorbidade } \\
\geq 2 \text { morbidades } \\
\% \text { (IC95\%) }\end{array}$} \\
\hline & $\begin{array}{l}\text { Sem morbidade } \\
\%(I C 95 \%)\end{array}$ & $\begin{array}{c}1 \text { morbidade } \\
\%(I C 95 \%)\end{array}$ & \\
\hline \multicolumn{4}{|l|}{$\begin{array}{l}\text { Sair de casa na última semana } \\
\text { (vezes na semana) }\end{array}$} \\
\hline Não saiu & $22,3(18,3-26,8)$ & $32,9(28,0-38,3)$ & $38,0(33,8-42,4)$ \\
\hline $1-2$ & $34,3(29,2-39,8)$ & $34,8(30,8-39,1)$ & $38,7(33,7-43,9)$ \\
\hline $3-5$ & $22,2(16,1-29,8)$ & $12,9(10,4-15,8)$ & $12,9(10,6-15,7)$ \\
\hline Quase todos os dias & $21,1(15,1-28,8)$ & $19,4(15,8-23,5)$ & $10,3(8,2-12,9)$ \\
\hline \multicolumn{4}{|l|}{ Motivo para sair de casa } \\
\hline \multicolumn{4}{|l|}{ Essenciais } \\
\hline $\begin{array}{l}\text { Comprar remédios ou } \\
\text { alimentos }\end{array}$ & $58,1(51,2-64,7)$ & $51,8(46,7-56,9)$ & $44,2(39,3-49,3)$ \\
\hline Trabalhar & $27,5(21,1-35,1)$ & $17,1(13,5-21,4)$ & $10,9(8,6-13,7)$ \\
\hline Pagar contas & $18,7(14,7-23,3)$ & $15,6(12,9-18,8)$ & $15,5(12,6-18,9)$ \\
\hline Obter atendimento de saúde & $4,4(2,9-6,5)$ & $5,5(3,8-8,1)$ & $9,3(7,5-11,5)$ \\
\hline \multicolumn{4}{|l|}{ Não essenciais } \\
\hline $\begin{array}{l}\text { Encontrar amigos ou } \\
\text { familiares }\end{array}$ & $9,1(5,3-15,3)$ & $5,8(3,9-8,4)$ & $4,7(3,4-6,5)$ \\
\hline $\begin{array}{l}\text { Fazer exercícios ou } \\
\text { caminhada }\end{array}$ & $5,5(2,9-10,1)$ & $4,0(2,6-5,9)$ & $3,5(2,4-5,0)$ \\
\hline Outra razão & $8,9(6,5-12,1)$ & $7,2(5,3-9,8)$ & $6,3(4,7-8,4)$ \\
\hline \multicolumn{4}{|l|}{ Uso de máscara ao sair de casa } \\
\hline Sempre & $96,6(94,2-97,9)$ & $96,9(95,1-98,1)$ & $98,1(96,8-98,9)$ \\
\hline Às vezes & $2,5(1,3-4,9)$ & $2,1(1,3-3,6)$ & $1,6(8,9-2,8)$ \\
\hline Nunca & $0,9(0,3-2,0)$ & $0,9(0,4-2,3)$ & $0,3(0,1-0,7)$ \\
\hline \multicolumn{4}{|l|}{$\begin{array}{l}\text { Lavar as mãos ou usar álcool } \\
\text { em gel }\end{array}$} \\
\hline Sempre & $96,7(93,3-98,4)$ & $97,3(95,7-98,3)$ & $97,8(96,0-98,8)$ \\
\hline Às vezes & $3,0(1,3-6,4)$ & $2,3(1,5-3,4)$ & $1,2(0,6-2,2)$ \\
\hline Nunca & $0,3(0,1-0,9)$ & $0,4(0,06-2,7)$ & $1,0(0,3-3,1)$ \\
\hline
\end{tabular}

IC95\%: intervalo de 95\% de confiança.

de morbidades. Entre aqueles que saíram de casa para realizar atividades essenciais, observa-se maior prevalência nas categorias de maior escolaridade (Figura 1a). As mulheres relataram ter ficado mais em casa do que os homens, no entanto, entre aqueles com multimorbidade, há uma diferença na prevalência de ter ficado em casa segundo sexo. Homens relataram sair para realizar atividades essenciais mais frequentemente do que mulheres, e tanto homens como mulheres com uma ou duas ou mais morbidades saíram menos para realizar atividades essenciais em relação àqueles sem nenhuma morbidade (Figura 1b). Para todas as categorias de classificação de morbidade, as faixas etárias mais avançadas relataram ficar mais em casa. Relação inversa foi observada para as atividades essenciais, em que as categorias mais jovens apresentam maiores prevalências de ter saído de casa, independente do número de morbidades (Figura 1c).

Quando analisamos os desfechos ajustados segundo características socioeconômicas foi possível observar maior adesão ao isolamento social (não sair de casa) entre as mulheres com multimorbidade, quando comparadas com os homens (RP = 1,49; IC95\%: 1,23-1,79). A adesão a esta medida aumentou proporcionalmente com a idade e inversamente ao nível de escolaridade (Tabela 3). 


\section{Figura 1}

Prevalência de medidas de proteção contra COVID-19 entre indivíduos com e sem multimorbidade, segundo escolaridade, sexo e faixa etária. Inquérito telefônico iniciativa ELSI-COVID-19, 2020.

1a) Escolaridade (anos)

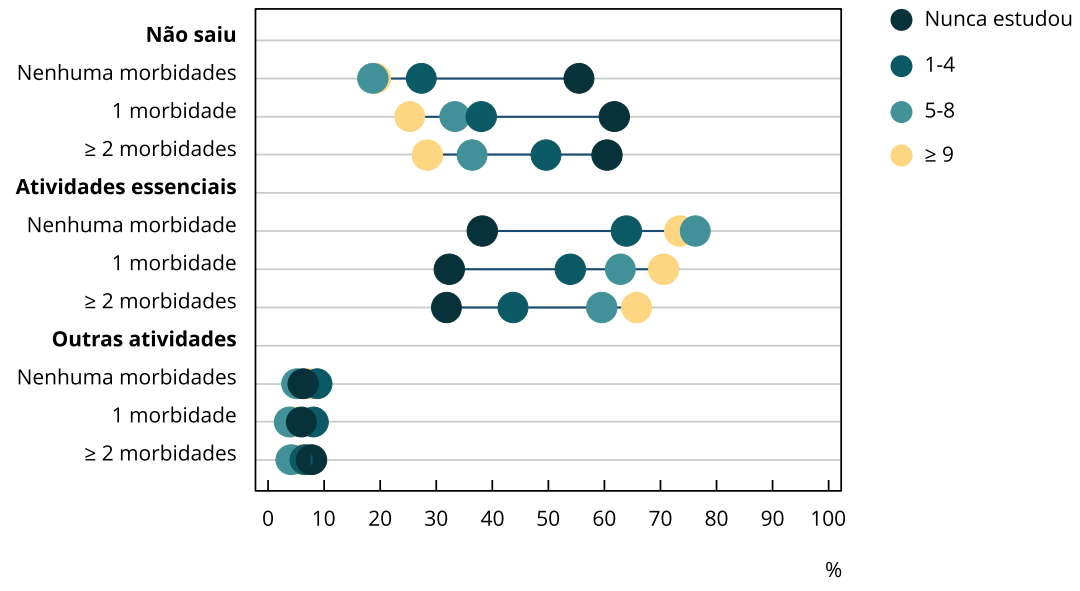

1b) Sexo

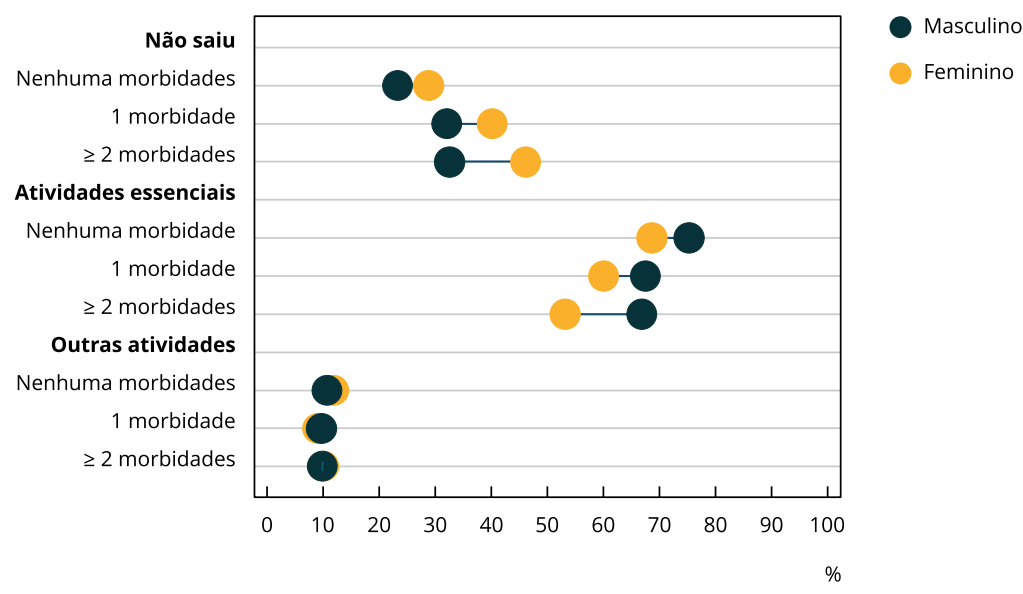

1c) Faixa etária (anos)

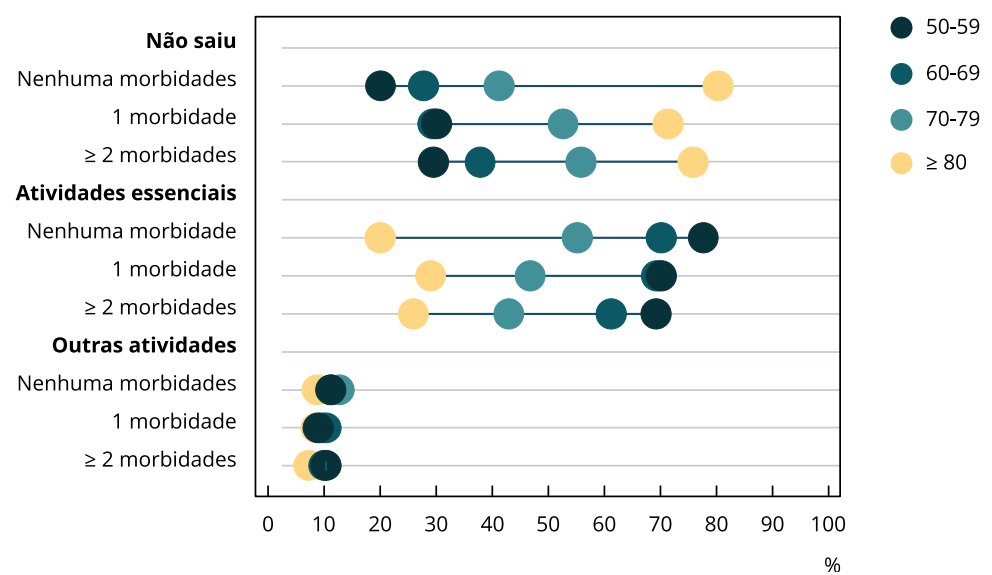


Tabela 3

Análise ajustada * entre não sair de casa e sexo, idade e escolaridade estratificada pelo número de morbidades. Inquérito telefônico iniciativa ELSI-COVID-19, 2020.

\begin{tabular}{|c|c|c|c|}
\hline \multirow[t]{3}{*}{ Variáveis } & \multicolumn{3}{|c|}{ Número de morbidades } \\
\hline & Sem morbidade & 1 morbidade & $\geq 2$ morbidades \\
\hline & RP (IC95\%) & RP (IC95\%) & RP (IC95\%) \\
\hline Sexo feminino [referência: homens] & $1,35(0,88-2,07)$ & $1,35(1,07-1,70)$ & $1,49(1,23-1,79)$ \\
\hline \multicolumn{4}{|l|}{ Idade (anos) [referência: 50-59] } \\
\hline $60-69$ & $1,55(0,92-2,62)$ & $1,00(0,74-1,35)$ & $1,32(1,03-1,70)$ \\
\hline $70-79$ & $2,06(1,12-3,79)$ & $1,60(1,19-2,14)$ & $1,99(1,56-2,53)$ \\
\hline$\geq 80$ & $4,02(2,50-6,47)$ & $2,36(1,78-3,14)$ & $2,64(1,94-3,58)$ \\
\hline \multicolumn{4}{|c|}{ Escolaridade (anos) [referência: nunca estudou] } \\
\hline $1-4$ & $0,68(0,48-0,97)$ & $0,76(0,65-0,90)$ & $1,02(0,86-1,21)$ \\
\hline $5-8$ & $0,61(0,39-0,95)$ & $0,79(0,60-1,05)$ & $0,84(0,72-0,99)$ \\
\hline$\geq 9$ & $0,61(0,38-0,98)$ & $0,57(0,41-0,79)$ & $0,71(0,56-0,90)$ \\
\hline
\end{tabular}

IC95\%: intervalo de 95\% de confiança; RP: razão de prevalência.

* Ajuste, por backward elimination em um nível hierárquico, para: sexo, idade, cor da pele, escolaridade, número de moradores, zona de residência e região geopolítica.

\section{Discussão}

A multimorbidade esteve associada com a adoção dos comportamentos atuais de proteção contra a COVID-19, principalmente isolamento social na semana anterior à entrevista. Pessoas com multimorbidade ficaram praticamente duas vezes mais em casa do que aquelas sem morbidades. Os principais motivos para sair de casa foram relacionados com atividades essenciais, e os mais frequentes (comprar remédios/alimentos e trabalhar) foram menos recorrentes em pessoas com multimorbidade. Somente sair para obter atendimento em saúde foi maior entre pessoas com multimorbidade, sinalizando a relevância da organização do sistema de saúde para atender a alta demanda de cuidados para pessoas com maior carga de morbidade. Mulheres, indivíduos com idade avançada e com menor nível de escolaridade saíram menos de casa em todas as categorias de morbidades. O uso de máscara e álcool em gel foi praticamente universal.

Na nossa amostra, a maioria dos indivíduos não saiu de casa ou saiu entre uma e duas vezes na última semana. É bastante plausível esperar que o grupo de pessoas mais idosas da população seja o que mais respeite as medidas de proteção, haja vista as evidências sobre o pior prognóstico entre idosos com SARS-CoV-2 e o enfoque claro dos governos em todo o mundo6. Todavia, estudo realizado na Malásia 27 mostra que, apesar de importante adesão dos cidadãos às medidas, esta foi menor naqueles com 50 anos ou mais e os de melhor renda. Nas Filipinas, 62,9\% dos entrevistados evitaram aglomerações 28 . Entre entrevistados alemães 29 , identificou-se que, embora fossem altos os níveis de conhecimento, a adoção de comportamentos importantes de proteção era muito baixa, e as percepções de risco eram especialmente baixas entre os idosos. Na Austrália 30 , manter-se afastado de locais lotados era geralmente o comportamento mais comum $(66,7 \%)$ e estiveram presentes quatro vezes mais naqueles que relataram estar preocupados com COVID-19 (alto-muito alto).

Contrapondo esses achados, um estudo baseado na análise de dados de 27 países de alta, média e baixa renda 18 demonstrou que as pessoas idosas não teriam maior probabilidade de isolamento em relação àquelas pessoas com 50 ou 60 anos. Para o autor, a avaliação entre todas as faixas etárias se revelou semelhante e com alta adesão às orientações sanitárias, não tendo efeito substancial com o aumento da idade. Revelou ainda que os mais idosos não são, necessariamente, mais responsivos em termos de autoisolamento prospectivo e vontade de isolar, além de não serem os mais disciplinados no cumprimento de medidas preventivas, especialmente quando usam máscara facial fora do domicílio. Por fim, como recomendação, a adoção de mecanismos de cuidado centrados no indi- 
víduo e de empatia pode ser de fundamental importância para a adesão às ações específicas entre os mais vulneráveis ${ }^{31}$.

Nosso estudo demonstrou maior adesão ao isolamento social (não sair de casa) entre as mulheres com multimorbidade em comparação aos homens, aumentando proporcionalmente com a idade e inversamente ao nível de escolaridade. A maior frequência de comportamento de proteção entre mulheres pode ser explicada pela maior conscientização sobre a importância de hábitos relacionados com a prevenção de doenças e promoção da saúde 32 . Ações como o fechamento de escolas para controlar a transmissão da COVID-19, como ocorreu na China, Hong Kong (SAR China), Itália, Coreia do Sul e também no Brasil, pode ter efeito diferencial sobre as mulheres, sobretudo por serem elas a fornecer a maior parte dos cuidados informais dentro das famílias, limitando seu trabalho e oportunidades econômicas 33. Durante o surto de 2014-2016 da doença do vírus ebola na África Ocidental, mulheres foram mais propensas a serem infectadas, em face dos seus papéis predominantes como cuidadoras dentro das famílias e como profissionais de saúde de linha de frente 34. A incorporação de análises de gênero na dimensão saúde, baseando-se inclusive na experiência de surtos passados, é fundamental para a efetividade das intervenções em saúde e promover equidade.

Indivíduos com multimorbidade saíram menos de casa em relação àqueles sem morbidade ( $38 \% \mathrm{e}$ $22,3 \%$, respectivamente), o que pode representar um dado importante no que tange às medidas protetivas, mas que também pode gerar dificuldades no controle de suas doenças e, possivelmente, gerar demanda às unidades de pronto atendimento devido à agudização dos problemas crônicos. $\mathrm{O}$ entendimento da razão para saída de casa entre pessoas com multimorbidade, ou seja, obter atendimento em saúde, precisa de discussão mais ampliada. Achar o equilíbrio entre as medidas de isolamento social e a necessidade de acompanhamento de sua situação de saúde pode ser um desafio para essa população, bem como para os seus familiares e os serviços de saúde 35. Neste sentido, parece fundamental fortalecer as ações de atenção primária à saúde para promover o cuidado adequado dos idosos perto de suas residências, utilizando-se estratégias de atenção a distância (quando possível) enquanto as estratégias de isolamento social forem necessárias 36,37,38.

Pessoas com menor escolaridade ficaram mais em casa, independente da presença de multimorbidade. Pesquisas anteriores 39 também mostraram que aqueles com maior renda estavam menos dispostos a cumprir as recomendações de saúde e percebiam menos medo e mais controle em situações de pandemia 40. A escolaridade representa um relevante indicador socioeconômico com impacto na saúde dos idosos, estando associada à maior ocorrência de multimorbidade $41 \mathrm{e}$, atualmente, isolamento social. Esse perfil pode ser prejudicial à saúde dos indivíduos caso as ações de cuidado, em especial de saúde mental 42 , não consigam atingir as pessoas em maior vulnerabilidade no atual contexto. Em síntese, anteriormente à pandemia, o perfil mais frequentemente acometido por multimorbidade era composto por mulheres, pessoas mais velhas e com menor escolaridade 10 , coincidindo com o perfil de pessoas que mais ficam em casa. Cuidar com foco nessas populações foi, é e será tarefa necessária para a busca da redução das iniquidades sociais em saúde. Para além dos "grupos de riscos" para infecção e prognóstico do SARS-CoV-2 (normalmente definidos como idosos e portadores de doenças crônicas), um olhar integral e humanizado do sistema de saúde e da sociedade ${ }^{8}$ para a população tem o potencial de melhor atender todas as demandas em saúde - históricas, presentes e futuras da pandemia - as quais são socialmente determinadas.

Dentre os participantes, foi observado que a maioria, quando saiu de casa, o fez por necessidade de comprar medicamentos ou alimentos, trabalhar e pagar contas. Cabe aqui lembrar que o medo de escassez das provisões essenciais, em idosos, é uma preocupação importante 43. Sair de casa todos os dias foi menos frequente naqueles indivíduos com multimorbidade, mas, entre estes, 9,3\% relataram ter saído de casa na última semana para obter atendimento de saúde.

A necessidade de se manterem os mais efetivos possíveis os tratamentos relacionados aos seus problemas de saúde é uma tônica, visto que já existem projeções de outras ondas, principalmente relacionadas à descompensação de problemas crônicos de saúde 35 . Em um estudo 30 , demonstrou-se que a grande maioria das pessoas poderia se autoisolar com suporte de familiar ou de amigo. Mesmo assim, tinham preocupações importantes relacionadas às dificuldades de compra de alimentos/suprimentos (48\%) e de acesso a serviços de saúde (39\%). Responsabilidades com crianças, pais idosos e familiares com deficiência foram os maiores impeditivos para aqueles que declararam não conseguir realizar autoisolamento em casa (8\%). 
De forma complementar, o fato de 9,1\% daqueles sem morbidade terem relatado visita a amigos e familiares por ocasião de saída de casa pode refletir o fato de que medidas de isolamento afetam de forma desproporcional os idosos. Geralmente, realizam de forma rotineira contato social fora de casa, frequentando centros comunitários ou locais religiosos. Pensar sobre essa necessidade, principalmente para as pessoas mais idosas, pode reduzir solidão, depressão e morbidade cardiovascular 44. Pessoas com 50 anos e mais têm, em média, menos da metade do número de contatos próximos em relação às pessoas com 18-29 anos, e esse valor reduz com o avançar da idade 45.

Naqueles indivíduos que saíram de casa na última semana, a utilização de máscaras faciais foi adotada pela grande maioria das pessoas (96\%) independente da presença de morbidades. Esse fato não foi observado na Malásia 27, onde quase 50\% relataram não utilizar. Cabe aqui lembrar que, na população malaia, assim como na brasileira, o uso de máscaras faciais não é um hábito rotineiro e que, no início da pandemia, alguns itens de proteção sofreram desabastecimento. Nas Filipinas, o uso foi relatado por $28 \%$ das pessoas, provavelmente refletindo os mesmos motivos da população malaia 28.

Entre os indivíduos com multimorbidade, a prevalência de não uso de máscaras faciais ou de usá-las às vezes é menor em relação àqueles sem ou com uma morbidade. Embora haja razoabilidade em predizer atitudes mais disciplinadas entre aqueles com maior risco de hospitalização e/ou morte por COVID-19, há indícios de que essa expectativa precisa ser revista 18 , principalmente quando se iniciar a diminuição de rigidez das medidas sanitárias. Estratégias de comunicação de práticas de risco e segurança efetivas para populações específicas, como idosos com multimorbidade, podem ser particularmente cruciais para a efetividade do cuidado em saúde.

A prevalência de lavagem das mãos ou uso de álcool em gel foi de, aproximadamente, 98\% na população em geral, sendo percentualmente maior entre aqueles com multimorbidade, seguindo dados de outros países 27,28. Em um estudo australiano, lavar as mãos com água e sabão foi o comportamento mais adotado relacionado à higiene (76,6\%). Verificou-se também relação importante entre preocupação com COVID-19 e maior envolvimento com essas medidas de proteção. A higienização das mãos representa uma estratégia facilmente adotável, dada à facilidade de compreensão, capacidade de envolvimento e disponibilidade de recursos para execução, além de ser o foco de comunicação de vários governos 30. Um estudo mostrou que 92\% de residentes dos Estados Unidos e Reino Unido adotariam comportamentos de higiene 46.

O presente estudo apresenta algumas limitações. As morbidades foram avaliadas por autorrelato de diagnóstico médico (exceto obesidade), o que tende a enviesar a real prevalência da multimorbidade. Considerando que, normalmente, esse viés tende a subestimar a prevalência do agravo, caso não existisse o erro, poderia ter sido observada uma maior diferença dos comportamentos segundo o número de doenças, por haver indivíduos com multimorbidade classificados com zero ou uma doença. Contudo, esse viés tende a ser pequeno, pois incluímos a maioria das morbidades crônicas mais prevalentes nesta população, e apenas 1,3\% da população de estudo relatou não saber ou não respondeu às perguntas sobre morbidades. Deve-se ainda considerar que a aferição dos comportamentos de precaução aqui estudados foram obtidos também por autorrelato, e não houve possibilidade metodológica de avaliação da qualidade dos mesmos. No referido inquérito, o isolamento foi considerado como o ato de não sair de casa, mas não se obtiveram informações sobre o recebimento de visitas em domicílio.

Neste contexto, novos desafios se fazem presentes a partir deste estudo. As pesquisas sobre a COVID-19 e seu impacto nos cidadãos e nos sistemas de saúde precisam ser inclusivas e socialmente responsáveis, envolvendo a população em envelhecimento. Deve, por esse motivo, abordar aspectos ampliados e relacionados à complexidade do cuidado da pessoa idosa, como questões relacionadas à multimorbidade, à fragilidade, ao declínio cognitivo e à moradia em Instituições de Longa Permanência para Idosos (ILPIs) 7,47,48. A relação da multimorbidade com as medidas de proteção contra a COVID-19 deve considerar a determinação social do processo saúde-doença no Brasil. Os fatores associados aos comportamentos de proteção têm potencial de afetar as pessoas com maior necessidade de cuidados. 


\section{Colaboradores}

S. R. Batista e B. P. Nunes participaram da concepção do manuscrito, escrita da primeira versão do artigo, revisão crítica do conteúdo e aprovou a versão final a ser publicada. A. S. S. Souza e J. Nogueira participaram da escrita da primeira versão do artigo, da análise dos dados, revisão crítica do manuscrito e aprovou a versão final a ser publicada. F. B. Andrade, E. Thumé, D. S. C. Teixeira, M. F. Lima-Costa e L. A. Facchini participaram da concepção do artigo, revisão crítica do manuscrito e aprovou a versão final a ser publicada. Todos os autores aprovaram a versão final a ser publicada e são responsáveis por todos os aspectos do trabalho, incluindo a garantia de sua precisão e integridade.

\section{Informações adicionais}

ORCID: Sandro Rodrigues Batista (0000-00017356-522X); Ana Sara Semeão de Souza (00000002-4554-1551); Januse Nogueira (0000-00015204-7116); Fabíola Bof de Andrade (0000-00023467-3989); Elaine Thumé (0000-0002-11698884); Doralice Severo da Cruz Teixeira (00000002-2894-3049); Maria Fernanda Lima-Costa (0000-0002-1077-1381); Luiz Augusto Facchini (0000-0002-5746-5170); Bruno Pereira Nunes (0000-0002-4496-4122).

\section{Referências}

1. World Health Organization. Coronavirus disease 2019 (covid-19). Situation report, 78. Geneva: World Health Organization; 2020.

2. Badawi A, Ryoo SG. Prevalence of comorbidities in the Middle East respiratory syndrome coronavirus (MERS-CoV): a systematic review and meta-analysis. Int J Infect Dis 2016; 49:129-33.

3. Banerjee A, Pasea L, Harris S, Gonzalez-Izquierdo A, Torralbo A, Shallcross L, et al. Estimating excess 1 -year mortality associated with the COVID-19 pandemic according to underlying conditions and age: a population-based cohort study. Lancet 2020; 395:1715-25.

4. Shahid Z, Kalayanamitra R, McClafferty B, Kepko D, Ramgobin D, Patel R, et al. Covid-19 and older adults: what we know. J Am Geriatr Soc 2020; 68:926-9.

5. Bello-Chavolla OY, González-Díaz A, Antonio-Villa NE, Fermín-Martínez CA, MárquezSalinas A, Vargas-Vázquez A, et al. Unequal impact of structural health determinants and comorbidity on COVID-19 severity and lethality in older Mexican adults: considerations beyond chronological aging. J Gerontol A Biol Sci Med Sci 2020; glaa163. [Online ahead of print].

\section{Agradecimentos}

O inquérito de base e a segunda onda do ELSI-Brasil foram financiados pelo Ministério da Saúde, Departamento de Ciência e Tecnologia da Secretaria de Ciência e Tecnologia e Insumos Estratégicos - DECIT/SCTI (404965/2012-1 e 28/2017) e Coordenação da Saúde da Pessoa Idosa - COSAPI/DAPES/SAS (20836, 22566, 23700 e 77/2019). A iniciativa ELSI-COVID-19 é financiada pelo DECIT/SCTI e pelo Conselho Nacional de Desenvolvimento Científico e Tecnológico (CNPq). M. F. Lima-Costa é bolsista IA de produtividade em pesquisa do CNPq. B. P. Nunes recebe financiamento do CNPq (Processo 432474/2016-1) e Fundação de Amparo à Pesquisa do Estado do Rio Grande do Sul (FAPERGS - 19/2551-0001231-4).
6. Jordan RE, Adab P, Cheng KK. Covid-19: risk factors for severe disease and death. BMJ 2020; 368:m1198.

7. Richardson SJ, Carroll CB, Close J, Gordon AL, O’Brien J, Quinn TJ, et al. Research with older people in a world with COVID-19: identification of current and future priorities, challenges and opportunities. Age Ageing 2020; afaa149.

8. Iaccarino G, Grassi G, Borghi C, Ferri C, Salvetti $M$, Volpe $M$, et al. Age and multimorbidity predict death among COVID-19 patients: results of the SARS-RAS Study of the Italian Society of Hypertension. Hypertension 2020; 76:366-72.

9. Nunes BP, Chiavegatto Filho ADP, Pati S, Cruz Teixeira DS, Flores TR, Camargo-Figuera FA, et al. Contextual and individual inequalities of multimorbidity in Brazilian adults: a crosssectional national-based study. BMJ Open 2017; 7:e015885.

10. Nunes BP, Batista SRR, Andrade FB, Souza Junior PRB, Lima-Costa MF, Facchini LA. Multimorbidity: the Brazilian Longitudinal Study of Aging (ELSI-Brazil). Rev Saúde Pública 2019; 52 Suppl 2:10s. 
11. Calderón-Larrañaga A, Vetrano DL, Ferrucci L, Mercer SW, Marengoni A, Onder G, et al. Multimorbidity and functional impairmentbidirectional interplay, synergistic effects and common pathways. J Intern Med 2019; 285:255-71

12. Guimarães RM, Andrade FCD. Healthy lifeexpectancy and multimorbidity among older adults: Do inequality and poverty matter? Arch Gerontol Geriatr 2020; 90:104157.

13. Oronce CIA, Scannell CA, Kawachi I, Tsugawa Y. Association between state-level income inequality and covid-19 cases and mortality in the USA. J Gen Intern Med 2020; 35:2791-3.

14. Bambra C, Riordan R, Ford J, Matthews F. The COVID-19 pandemic and health inequalities. J Epidemiol Community Health 2020; 74:964-8.

15. Kalanche A. Coronavirus makes inequality a public health issue. World Economic Forum, 2020. https://www.weforum.org/ agenda/2020/04/coronavirus-makes-inequal ity-a-public-health-issue/ (acessado em 05/ $\mathrm{Jul} / 2020)$.

16. Baqui P, Bica I, Marra V, Ercole A, van der Schaar M. Ethnic and regional variations in hospital mortality from COVID-19 in Brazil: a cross-sectional observational study. Lancet Glob Health 2020; 8:e1018-e1026.

17. Hallal P, Hartwig F, Horta B, Victora GD, Silveira $M$, Struchiner C, et al. Remarkable variability in SARS-CoV-2 antibodies across Brazilian regions: nationwide serological household survey in 27 states. medRxiv 2020; 30 mai. https://www.medrxiv.org/content/10.11 01/2020.05.30.20117531v1.

18. Daoust J-F. Elderly people and responses to Covid-19 in 27 Countries. PLoS One 2020; 15:e0235590.

19. Utych SM, Fowler L. Age-based messaging strategies for communication about Covid-19. J Behav Public Adm 2020; 3(1). https://jour nal-bpa.org/index.php/jbpa/article/view/151.

20. Mesa Vieira C, Franco OH, Gómez Restrepo C, Abel T. Covid-19: the forgotten priorities of the pandemic. Maturitas 2020; 136:38-41.

21. Brasil. Ministério da Saúde. Portaria no 454, de 20 de março de 2020. Declara, em todo o território nacional, o estado de transmissão comunitária do coronavírus (COVID-19). Diário Oficial da União 2020; 20 mar.

22. Lima-Costa MF, Andrade FB, Souza Jr. PRB, Neri AL, Duarte YAO, Castro-Costa E, et al. The Brazilian Longitudinal Study of Aging (ELSI-Brazil): objectives and design. Am J Epidemiol 2018; 187:1345-53.

23. Lima-Costa MF, Macinko J, Andrade FB, Souza Jr. PRB, Vasconcellos MTL, Oliveira CM. ELSI-COVID-19 initiative: methodology of the telephone survey on coronavirus in the Brazilian Longitudinal Study of Aging. Cad Saúde Pública 2020; 36 Suppl 3:e00183120.
24. Clark A, Jit M, Warren-Gash C, Guthrie B, Wang $\mathrm{HH}$, Mercer SW, et al. How many are at increased risk of severe COVID-19 disease? Rapid global, regional and national estimates for 2020. medRxiv 2020; $22 \mathrm{abr}$. https://www. medrxiv.org/content/10.1101/2020.04.18.200 $64774 \mathrm{v} 1$.

25. Lippi G, Mattiuzzi C, Sanchis-Gomar F, Henry BM. Clinical and demographic characteristics of patients dying from COVID-19 in Italy vs China. J Med Virol 2020; 10.1002/jmv.25860.

26. World Health Organization. Obesity: preventing and managing the global epidemic. http:// www.who.int/entity/nutrition/publications/ obesity/WHO_TRS_894/en/index.html (acessado em 05/Jul/2020).

27. Azlan AA, Hamzah MR, Sern TJ, Ayub SH, Mohamad E. Public knowledge, attitudes and practices towards COVID-19: a crosssectional study in Malaysia. PLoS One 2020; 15:e0233668.

28. Lau LL, Hung N, Go DJ, Ferma J, Choi M, Dodd W, et al. Knowledge, attitudes and practices of COVID-19 among income-poor households in the Philippines: a cross-sectional study. J Glob Health 2020; 10:011007.

29. Betsch C. How behavioural science data helps mitigate the COVID-19 crisis. Nat Hum Behav 2020; 4:438.

30. Seale H, Heywood AE, Leask J, Sheel M, Thomas S, Durrheim DN, et al. COVID-19 is rapidly changing: examining public perceptions and behaviors in response to this evolving pandemic. Tu W-J, editor. PLoS One 2020; 15:e0235112.

31. Pfattheicher S, Nockur L, Böhm R, Sassenrath $\mathrm{C}$, Petersen MB. The emotional path to action: Empathy promotes physical distancing and wearing face masks during the COVID-19 pandemic. PsyArXiv 2020; 23 mar. https:// psyarxiv.com/y2cg5/.

32. Brito A, Camargo B. Representações sociais, crenças e comportamentos de saúde: um estudo comparativo entre homens e mulheres. Temas Psicol 2011; 9:282-303.

33. Wenham C, Smith J, Morgan R. Covid-19: the gendered impacts of the outbreak. Lancet 2020; 395:846-8.

34. Davies SE, Bennett B. A gendered human rights analysis of Ebola and Zika: locating gender in global health emergencies. Int Aff 2016; 92:1041-60.

35. Kohli P, Virani SS. Surfing the waves of the COVID-19 pandemic as a cardiovascular clinician. Circulation 2020;142:98-100.

36. Daumas RP, Silva GA, Tasca R, Leite IC, Brasil P, Greco DB, et al. O papel da atenção primária na rede de atenção à saúde no Brasil: limites e possibilidades no enfrentamento da COVID-19. Cad Saúde Pública 2020; 36:e00104120. 
37. Greenhalgh T, Choon Huat Koh G, Car J. Covid-19: avaliação remota em atenção primária à saúde. Rev Bras Med Fam Comunidade 2020; 15:2461.

38. Sarti TD, Lazarini WS, Fontenelle LF, Almeida APSC. Qual o papel da Atenção Primária à Saúde diante da pandemia provocada pela COVID-19? Epidemiol Serv Saúde 2020; 29:e2020166.

39. Wong KK, Cohen AL, Norris SA, Martinson NA, von Mollendorf C, Tempia S, et al. Knowledge, attitudes, and practices about influenza illness and vaccination: a cross-sectional survey in two South African communities. Influenza Other Respir Viruses 2016; 10:421-8.

40. Raude J, Setbon M. Lay perceptions of the pandemic influenza threat. Eur J Epidemiol 2009; 24:339-42.

41. Pathirana TI, Jackson CA. Socioeconomic status and multimorbidity: a systematic review and meta-analysis. Aust N Z J Public Health 2018; 42:186-94.

42. Fiorillo A, Gorwood P. The consequences of the COVID-19 pandemic on mental health and implications for clinical practice. Eur Psychiatry 2020; 63:e32.
43. Lloyd-Sherlock P, Ebrahim S, Geffen L, McKee M. Bearing the brunt of covid-19: older people in low and middle income countries. BMJ 2020; 368:m1052.

44. Berg-Weger M, Morley JE. Loneliness in old age: an unaddressed health problem. J Nutr Health Aging 2020; 24:243-5.

45. Canning D, Karra M, Dayalu R, Guo M, Bloom DE. The association between age, COVID-19 symptoms, and social distancing behavior in the United States. medRxiv 2020; 23 abr. https://www.medrxiv.org/content/10.1101/2 020.04.19.20065219v1.

46. Geldsetzer P. Use of rapid online surveys to assess people's perceptions during infectious disease outbreaks: a cross-sectional survey on COVID-19. J Med Internet Res 2020; 22:e18790.

47. Aprahamian I, Cesari M. Geriatric syndromes and SARS-Cov-2: more than just being old. J Frailty Aging 2020; 9:127-9.

48. Chan EYY, Gobat N, Kim JH, Newnham EA, Huang $\mathrm{Z}$, Hung $\mathrm{H}$, et al. Informal home care providers: the forgotten health-care workers during the COVID-19 pandemic. Lancet 2020; 395:1957-9. 


\section{Abstract}

To measure the occurrence of protective behaviors for COVID-19 and sociodemographic factors according to the occurrence of multimorbidity in the Brazilian population aged 50 or over was the objective of this study. We used data from telephone surveys among participants of ELSI-Brazil (Brazilian Longitudinal Study of Aging), conducted between May and June 2020. The use of non-pharmacological prevention measures for COVID-19, reasons for leaving home according to the presence of multimorbidity and sociodemographic variables were evaluated. among 6,149 individuals. Multimorbidity was more frequent in females, married, aged 50-59 years and residents of the urban area. Most of the population left home between once and twice in the last week, increasing according to the number of morbidities $22.3 \%$ no morbidities and $38 \%$ with multimorbidity). Leaving home every day was less common among individuals with multimorbidity (10.3\%) and 9.3\% left home in the last week to access health care. Hand hygiene (>98\%) and always wearing a mask when leaving home (>96\%) were almost universal habits. Greater adherence to social isolation was observed among women with multimorbidity when compared to men (PR $=1.49$, 95\%CI: 1.23-1.79). This adherence increased proportionally with age and inversely with the level of education. The protective behavior in people with multimorbidity seems to be greater in relation to the others, although issues related to social isolation and health care deserve to be highlighted. These findings can be useful in customizing strategies for coping with the current pandemic.

Multimorbidity; COVID-19; Behavior; SAR-CoV-2; Chronic Disease

\section{Resumen}

El objetivo fue medir la ocurrencia de comportamientos de protección contra la COVID-19y factores sociodemográficos, según la ocurrencia de multimorbilidad, en la población brasileña con 50 años o más de edad. Se utilizaron datos de la encuesta telefónica entre participantes del ELSI- Brasil (Estudio Brasileño Longitudinal del Envejecimiento), realizado entre mayo y junio de 2020. Se evaluó el uso de medidas de prevención no farmacológica para la COVID-19, motivos para salir de casa, según la presencia de multimorbilidad y variables sociodemográficas. Participaron del estudio 6.149 personas. La multimorbilidad fue más frecuente en el sexo femenino, en casados, en la franja de edad 50-59 años de edad y en residentes de la zona urbana. La mayor parte de la población salió de casa entre una y dos veces en la última semana, porcentaje que aumentó según el número de morbilidades (22,3\% sin morbilidades $y$ $38 \%$ con multimorbilidad). Salir de casa todos los días tuvo una menor ocurrencia entre individuos con multimorbilidad (10,3\%), y 9,3\% salieron de casa en la última semana para obtener atención en salud. La higienización de manos (> 98\%) y siempre usar mascarilla al salir de casa (> 96\%) fueron hábitos casi universales. Se observó una mayor adhesión al aislamiento social entre las mujeres con multimorbilidad cuando se compararon con los hombres ( $R P=1,49$; IC95\%: 1,23-1,79); esta adhesión aumentó proporcionalmente con la edad y fue inversamente proporcional al nivel de escolaridad. El comportamiento de protección en personas con multimorbilidad parece ser mayor respecto a los demás, a pesar de que las cuestiones relacionadas con el aislamiento social y cuidado en salud merezcan ser destacadas. Estos resultados pueden ser útiles en la personalización de estrategias de combate a la actual pandemia.

Multimorbidad; COVID-19; Conducta; SAR-CoV-2; Enfermedad Crónica
Recebido em 07/Jul/2020

Versão final reapresentada em 27/Jul/2020

Aprovado em 07/Ago/2020 